\title{
Stabilization of an Anion Radical Formed in Poly(vinyl methyl terephthalate) Studied by Anion Radical Transfer Method
}

\author{
Yoshinobu TsujII, Akira Tsuchida, Masahide Yamamoto ${ }^{\dagger}$ \\ Yasunori NISHIJIMA, and Yoshio WADA* \\ Department of Polymer Chemistry, Faculty of Engineering, \\ Kyoto University, Yoshida, Sakyo-ku, Kyoto 606, Japan \\ * Department of Photographic Engineering, Kyoto Institute of Technology, \\ Matsugasaki, Sakyo-ku, Kyoto 606, Japan
}

(Received March 17, 1988)

\begin{abstract}
Photoexcitation of $N$-ethylcarbazole (donor) in the presence of a polymer having terephthalate chromophores (acceptor) in solution produces a terephthalate anion radical in the polymer. The stability of the terephthalate anion radical $\left(\mathrm{TP}^{-}\right)$in the polymer chain was investigated by the anion radical transfer method using nanosecond laser photolysis. The samples were poly(vinyl methyl terephthalate) (PVMTP), copolymers of vinyl methyl terephthalate (VMTP) with vinyl acetate (VAc), and the monomer model compound, dimethyl terephthalate (DMTP). The anion radical $\mathrm{TP}^{-}$formed in the homopolymer, PVMTP does not transfer an electron to 1,4dicyanobenzene (DCNB), but the monomer model compound, DMTP does, although the two systems have the same transient absorption spectrum. As for the anion radical $\mathrm{TP}^{-}$in the copolymer, the anion radical transfer to DCNB has two components, a fast and a slow component: the fast component has a rate constant of $7.7 \times 10^{8} \mathrm{M}^{-1} \mathrm{~s}^{-1}$ and the slow component is extremely slow. The fraction of the slow component increases with the VMTP fraction. The results show that the neighboring interactions of TP residues in the polymer chain stabilize the anion radical of the TP residue. The anion radical $\mathrm{TP}^{-}$formed in the polymer is stabilized partly due to neighboring interactions and partly due to the steric hindrance of the polymer chain to an approaching DCNB.

KEY WORDS Stabilization / Neighboring Chromophore Interaction /

Anion Radical / Anion Radical Transfer / Laser Photolysis / Poly(vinyl methyl terephthalate) / Poly(vinyl methyl terephthalate-co-vinyl acetate) / Dicyanobenzene /
\end{abstract}

In photophysical and photochemical processes in polymers having chromophores as pendant groups, interactions between neighboring chromophores play important roles. Recently extensive investigations have been made on interactions between an excited chromophore and a ground state one (excimer and exciplex) or between an ion radical chromophore and a neutral one (dimer ion radical). ${ }^{1-6}$

\footnotetext{
† To whom all correspondence should be addressed.
}

As for excited chromophores in polymers, the excitation energy migrates efficiently along the polymer chain, ${ }^{7-9}$ and an intramolecular excimer is formed through energy migration. ${ }^{10,11}$ Annihilation of excited states also occurs frequently through energy migration under a high photon-density excitation such as laser excitation. ${ }^{12,13}$

A dimer ion radical is formed by interactions between an ion radical chromophore and 
an adjacent ground state chromophore. The properties of cation radicals formed in polymers and in the dimer model compounds have been investigated using $\mathrm{ESR},{ }^{14,15}$ CIDNP ${ }^{16}$ gamma-ray experiment, ${ }^{17-20}$ pulse radiolysis, ${ }^{21,22}$ and laser photolysis. $^{23,24}$ Recently, we reported the stabilization of the cation radical formed photochemically in poly $(N$-vinylcarbazole) $(\mathrm{PVCz})$ and its dimer model compounds (1,3-di( $N$-carbazolyl)propane and 2,4-di( $N$-carbazolyl)pentanes). We used the cation radical transfer method with nanosecond laser photolysis, and estimated quantitatively the stabilization energy of the dimer cation radical for the dimer model compounds. ${ }^{25}$ On the other hand, there are only a few reports about the formation of aromatic dimer anion radicals. ${ }^{26,27}$ Recently, we reported the stabilization of the anion radical formed in poly(vinyl methyl terephthalate) (PVMTP) and its dimer model compounds by the anion radical transfer method. ${ }^{28}$ For both PVMTP and the dimer model compounds, where the terephthalate groups are linked by a methylene chain, the anion radical transfer to 1,4-dicyanobenzene (DCNB) or to 1,2,4,5-tetracyanobenzene (TCNB) was found to be suppressed in comparison with a monomer model compound, dimethyl terephthalate (DMTP). Furthermore the suppression for PVMTP was much stronger than that for the dimer model compounds and no anion radical transfer to DCNB could be observed for PVMTP. This is considered due to the stabilization of the anion radical by interactions with the neighboring chromophores.

In this study, we investigated the anion radical transfer to DCNB using nanosecond laser photolysis for the copolymer of vinyl methyl terephthalate (VMTP) with vinyl acetate (VAc). The relation between the sequence of TP chromophores and the stabilization was studied in detail to elucidate the behavior of the anion radical formed in PVMTP.

\section{EXPERIMENTAL}

\section{Copolymers}

Vinyl methyl terephthalate (VMTP) was synthesized by the ester exchange reaction of vinyl acetate with terephthalic acid monomethyl ester (Tokyo Kasei Kogyo Co.) ${ }^{29}$ and was purified by a silica-gel column chromatography with the eluent of dichloromethane and by recrystallization from hexane. Vinyl acetate (VAc, Wako Pure Chem. Ind., Ltd.) was purified by distillation. Four samples of the copolymer of VMTP with VAc were prepared by a radical copolymerization initiated by AIBN in a benzene solvent at $60^{\circ} \mathrm{C}$ and their conversions of the copolymerization were suppressed to below $30 \mathrm{~mol} \%$. They were purified by precipitation three times from a benzene solution into hexane. The molecular weight $\left(\bar{M}_{w}\right)$ of these copolymers was measured by GPC (Toyo Soda HLC 802 UR) with a $\mathrm{G} 4000 \mathrm{H}$ and a GMH6 column. The fraction of terephthalate units $\left(f_{\mathrm{TP}}\right)$ in these copolymers was determined by UV absorption spectra on the basis of the molar extinction coefficient of dimethyl terephthalate (DMTP, $\varepsilon=1.87 \times 10^{3}$ $\mathrm{M}^{-1} \mathrm{~cm}^{-1}$ at $286 \mathrm{~nm}$ ) in dichloromethane. These data are shown in Table I with the feed ratios. These copolymers were designated as P1, P2, P3, and P4 in the order of increasing $f_{\mathrm{TP}}$. The monomer reactivity ratios, $r_{1}$ and $r_{2}$, were determined by the Fineman-Ross

Table I. Characterization of copolymers of VMTP with VAc

\begin{tabular}{cllc}
\hline Copolymer & $F^{\mathrm{a}}$ & $f_{\mathrm{TP}}{ }^{\mathrm{b}}$ & $\bar{M}_{w} / 10^{4 \mathrm{c}}$ \\
\hline P1 & 0.20 & 0.18 & 1.9 \\
P2 & 0.52 & 0.31 & 2.9 \\
P3 & 1.2 & 0.49 & 3.4 \\
P4 & 3.5 & 0.62 & 3.4 \\
\hline
\end{tabular}

a Monomer feed ratio of the copolymerization $(F=$ $[\text { VMTP }]_{0} /[\mathrm{VAc}]_{0}$ ).

b Mole fraction of TP unit in the copolymer determined by UV absorption.

c Molecular weights determined by GPC (polystyrene equivalent). 
method $^{30}$ to be 0.26 and 0.64 , respectively.

\section{Other Chemicals}

$N$-Ethylcarbazole (EtCz) was synthesized by $N$-alkylation of carbazole and was purified by recrystallization. Dimethyl terephthalate (DMTP, Wako Pure Chem. Ind., Ltd.) and 1,4-dicyanobenzene (DCNB, Wako Pure Chem. Ind., Ltd.) were purified several times by recrystallization. Dimer model compounds, 1,3-bis[ $p$-(methoxycarbonyl)propane] (1,3MTP) and 2,4-bis[ $p$-(methoxycarbonyl)pentane] $(2,4-\mathrm{MTP})$ were synthesized and purified as reported previously. ${ }^{28} \mathrm{~A}$ racemic isomer of two diastereoisomers was used as 2,4MTP. Dichloromethane (Dotite Spectrosol) was used without further purification. Benzonitrile (Wako Pure Chem. Ind., Ltd.) was fractionally distilled.

\section{Measurements}

When an electron donor, $\mathrm{EtCz}$, is excited in the presence of an electron acceptor, a TP chromophore in benzonitrile, a cation radical of $\mathrm{EtCz}\left(\mathrm{EtCz}^{+}\right)$and an anion radical of TP $\left(\mathrm{TP}^{-}\right)$are produced by the electron transfer. These ion radicals decay by recombination (eq 1). Then, the addition of an anion radical acceptor, DCNB, which has a lower reduction potential than the TP chromophore, causes the anion radical transfer (an electron transfer) from $\mathrm{TP}^{-}$to $\mathrm{DCNB}$ and an anion radical of DCNB $\left(\mathrm{DCNB}^{-}\right)$is produced (eq 2). The formed $\mathrm{DCNB}^{-}$also decays by recombination with $\mathrm{EtCz}^{+}$(eq 3).

$$
\begin{aligned}
& \mathrm{EtCz}^{+}+\mathrm{TP}^{-} \rightarrow \mathrm{EtCz}+\mathrm{TP} \\
& \mathrm{TP}^{-}+\mathrm{DCNB}^{-\mathrm{TP}}+\mathrm{DCNB}^{-} \\
& \mathrm{EtCz}^{+}+\mathrm{DCNB}^{-} \rightarrow \mathrm{EtCz}^{-} \mathrm{DCNB}^{-}
\end{aligned}
$$

The absorption spectra and decays of $\mathrm{EtCz}^{+}$, $\mathrm{TP}^{-}$, and $\mathrm{DCNB}^{-}$were measured by nanosecond laser photolysis. Selective photoexcitation of $\mathrm{EtCz}$ was made by a second harmonic pulse $(347 \mathrm{~nm}, 10 \mathrm{~mJ}, 14 \mathrm{~ns})$ of a Q-switched giant pulse ruby laser (NEC SLG2009) for copolymer systems and by an excimer laser pulse of XeF (Lambda Physik EMG101MSC, $351 \mathrm{~nm}, 60 \mathrm{~mJ}, 18 \mathrm{~ns}$ ) for model compound systems. All samples for the laser photolysis were degassed by the freeze-pump-thaw method. In the measurement of the laser photolysis, the absorbance of $\mathrm{EtCz}$ was adjusted to about unity at an excitation wavelength and the TP chromophore concentration was adjusted to $1.0 \times 10^{-1} \mathrm{M}$ for copolymers and to $5.0 \times 10^{-2} \mathrm{M}$ for model compounds. For the anion radical transfer experiments, $1.0 \times$ $10^{-2} \mathrm{M}$ DCNB for copolymer systems and $2.0 \times 10^{-3} \mathrm{M}$ DCNB for model compound systems were added to the above systems.

The UV absorption spectra of these copolymers in dichloromethane were measured by a Shimadzu UV-200s spectrometer.

The reduction potentials of DMTP and DCNB were measured by cyclic voltammetry with the reference electrode $\left(\mathrm{Ag} / 0.01 \mathrm{~N} \mathrm{Ag}^{+}\right.$in acetonitrile) and were determined to be $-2.11 \mathrm{~V}$ and $-1.97 \mathrm{~V}$, respectively.

\section{RESULTS AND DISCUSSION}

\section{Transient Absorption Spectra}

Figure 1 shows the transient absorption spectrum of the EtCz-P1 system in benzoni-

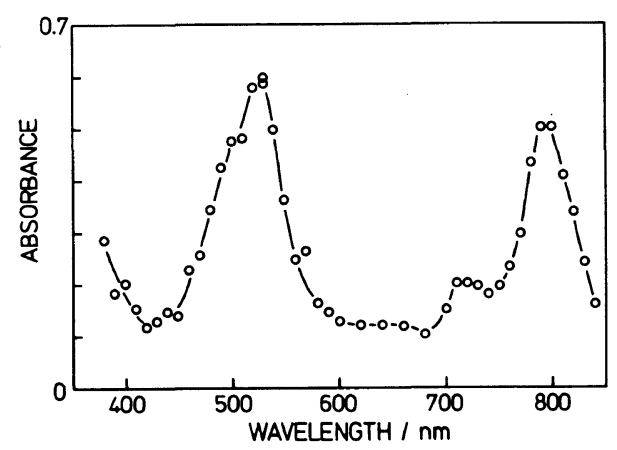

Figure 1. Transient absorption spectrum of $\mathrm{EtCz}-\mathrm{Pl}$ system in benzonitrile measured by nanosecond laser photolysis at $298 \mathrm{~K}$ at $1 \mu \mathrm{s}$ after excitation. The absorbance of $\mathrm{EtCz}$ at $347 \mathrm{~nm}$ is about unity. The concentration of TP chromophore in the copolymer is $1.0 \times 10^{-1} \mathrm{M}$. 


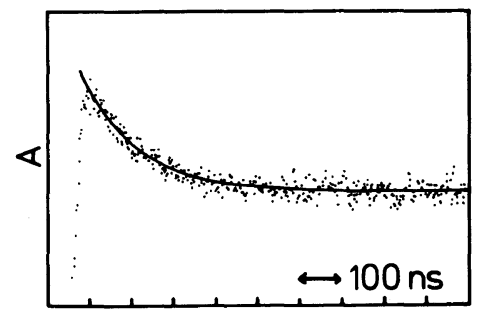

(I)

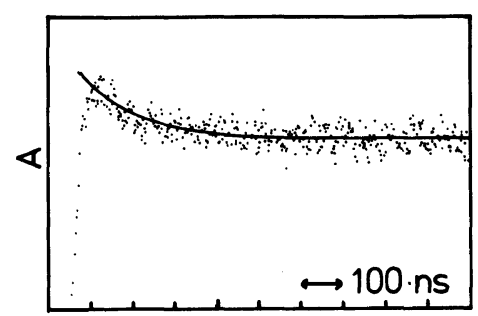

(III)

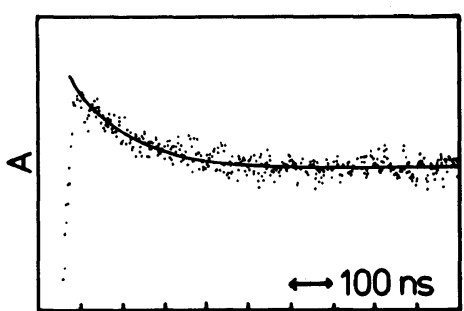

(II)

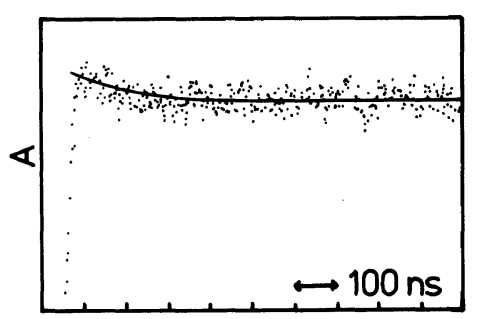

(IV)

Figure 2. Transient absorbance decay measured at $530 \mathrm{~nm}$ in benzonitrile. (I), EtCz-P1-DCNB system; (II), EtCz-P2-DCNB system; (III), EtCz-P3-DCNB system; (IV), EtCz-P4-DCNB system. The absorbance of $\mathrm{EtCz}$ is about unity. The concentrations of TP chromophore in the copolymers and DCNB in each system are $1.0 \times 10^{-1} \mathrm{M}$ and $1.0 \times 10^{-2} \mathrm{M}$, respectively.

trile. This spectrum is almost the same as that of the monomer model compound, $\mathrm{EtCz}-$ DMTP system. The absorption bands around $780 \mathrm{~nm}$ and $530 \mathrm{~nm}$ are assigned to $\mathrm{EtCz}^{+}$and $\mathrm{TP}^{-}$in the copolymer, respectively. Other copolymer systems give similar transient absorption spectra as that shown in Figure 1. The molar extinction coefficient of $\mathrm{TP}^{-}$for each copolymer at $530 \mathrm{~nm}$ was determined to be $c a$. $1.3 \times 10^{4} \mathrm{M}^{-1} \mathrm{~cm}^{-1}$ on the basis of that of $\mathrm{EtCZ}^{+}$at $780 \mathrm{~nm}\left(\varepsilon=9.4 \times 10^{3} \mathrm{M}^{-1} \mathrm{~cm}^{-1}\right)$. The molar extinction coefficient of $\mathrm{TP}^{-}$in the copolymer was independent of $f_{\mathrm{TP}}$. This value is almost the same as that of DMTP ${ }^{-}(\varepsilon=$ $1.2 \times 10^{4} \mathrm{M}^{-1} \mathrm{~cm}^{-1}$ ). The fact that no change was observed in the transient absorption spectra means weak interactions between the neighboring chromophores.

\section{Anion Radical Transfer from $T P^{-}$to DCNB}

Figure 2 shows the decay at $530 \mathrm{~nm}$ which was mainly assigned to $\mathrm{TP}^{-}$in the copolymer. In the absence of $\mathrm{DCNB}, \mathrm{TP}^{-}$decays by the recombination with $\mathrm{EtCz}^{+}$, but its decay is

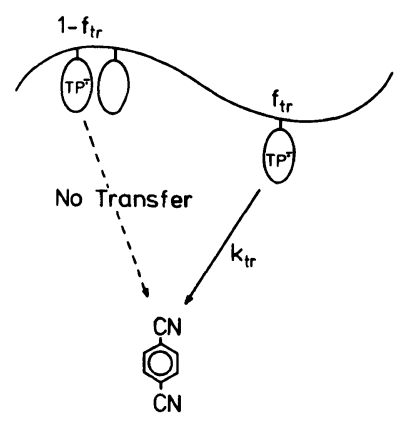

Figure 3. Scheme of anion radical transfer from $\mathrm{TP}^{-}$ in copolymers to DCNB.

hardly observed in this time region $(<1 \mu \mathrm{s})$. On the contrary, addition of DCNB to this system accelerates the decrease of $\mathrm{TP}^{-}$as shown in Figure 2. This is due to the anion radical transfer from $\mathrm{TP}^{-}$in the copolymer to DCNB. The decay consists of a fast decay component in the short time region $(<c a$. $200 \mathrm{ns)}$ and a slow decay component after the fast decay. As $f_{\mathrm{TP}}$ increases from 0.18 to 0.62 , the fast decay component decreases.

We simulated these decay curves using the 
scheme shown in Figure 3. One part of $\mathrm{TP}^{-}$in the copolymer transfers an electron to DCNB in the rate constant $k_{\mathrm{tr}}$ with a fraction of $f_{\mathrm{tr}}$ and the others that are stabilized by the neighboring interaction do not. The concentration of $\mathrm{TP}^{-}\left(\left[\mathrm{TP}^{-}\right]\right)$at time $t$ is expressed by eq 4 , as the decrease of $\mathrm{TP}^{-}$by the recombination with $\mathrm{EtCz}^{+}$is negligible in this time region $(<1 \mu \mathrm{s})$.

$$
\begin{aligned}
{\left[\mathrm{TP}^{-}\right]=} & {\left[\mathrm{TP}^{-}\right]_{0}\left(f_{\mathrm{tr}} \exp \left(-k_{\mathrm{tr}}[\mathrm{DCNB}] t\right)\right.} \\
& \left.+\left(1-f_{\mathrm{tr}}\right)\right)
\end{aligned}
$$

where $\left[\mathrm{TP}^{-}\right]_{0}$ and $[\mathrm{DCNB}]$ are the concentration of $\mathrm{TP}^{-}$and DCNB at $t=0$, respectively. The first term in the parentheses corresponds to the fast decay component in the observed decay curve and represents the decrease through the anion radical transfer. The second term corresponds to the slow decay component which does not decrease in this time region.

The observed decay curves at $530 \mathrm{~nm}$ were simulated using eq 4. As the absorbances of $\mathrm{EtCz}^{+}$and $\mathrm{DCNB}^{-}$overlap slightly on that of $\mathrm{TP}^{-}$, the contribution of these ion radicals was subtracted on simulation. The initial concentration $\left[\mathrm{TP}^{-}\right]_{0}$ was also determined from the absorbance at $t=0$ at $530 \mathrm{~nm}$ in consideration of the overlap contribution of $\mathrm{EtCz}^{+}$and $\mathrm{DCNB}^{-}$. The formation of $\mathrm{DCNB}^{-}$through direct quenching of an excited $\mathrm{EtCz}$ was also taken into account. The results are summarized in Table II and the simulation lines are given by the solid line in Figure 2. The rate constant $k_{\mathrm{tr}}$ was found to be $7.7 \times 10^{8} \mathrm{M}^{-1} \mathrm{~s}^{-1}$ for each system. The fraction of electrontransferable TP, $f_{\text {tr }}$ decreases in the order, P1

Table II. Simulation parameters of decay curves at $530 \mathrm{~nm}$

\begin{tabular}{ccc}
\hline Copolymer & $f_{\mathrm{tr}}$ & $k_{\mathrm{tr}} / 10^{8} \mathrm{M}^{-1} \mathrm{~s}^{-1}$ \\
\hline P1 & 0.83 & 7.7 \\
P2 & 0.64 & 7.7 \\
P3 & 0.48 & 7.7 \\
P4 & 0.21 & 7.7 \\
\hline
\end{tabular}

$\left(f_{\mathrm{tr}}=0.83\right), \mathrm{P} 2\left(f_{\mathrm{tr}}=0.64\right), \mathrm{P} 3\left(f_{\mathrm{tr}}=0.48\right)$, and $\mathrm{P} 4\left(f_{\mathrm{tr}}=0.21\right)$ with the increase of $f_{\mathrm{TP}}$.

\section{Interactions of Neighboring Chromophores in the Copolymer}

In this system, the degree of interactions between the neighboring chromophores depends on the sequence distribution of VMTP in the copolymer. The fraction $\left(f_{\mathrm{i}}\right)$ that the TP group is isolated in the copolymer, that is, the fraction of the sequence, VAc-VMTP-VAc was calculated from $r_{1}$ and the feed ratio $(F=$ [VMTP]/[VAc]) using eq $5 .^{31}$

$$
f_{i}=\left(1+r_{1} F\right)^{-2}
$$

The calculated values $f_{i}$ of P1, P2, P3, and P4 are $0.90,0.78,0.58$, and 0.27 , respectively. Figure 4 shows the relation between the transferable fraction $f_{\mathrm{tr}}$ and the calculated value $f_{i}$. The value $f_{\mathrm{tr}}$ is somewhat smaller than $f_{i}$ for each copolymer. One reason for the difference is considered due to the underestimation of the initial absorbance of $\mathrm{TP}^{-}$: the anion radical transfer occurs within the excitation pulse width of $14 \mathrm{~ns}$. It is concluded that the transferable fraction $f_{\text {tr }}$ is roughly proportional to $f_{i}$. This means that the electron-transferable anion radical $\mathrm{TP}^{-}$is the one formed in the sequence, VAc-VMTP-VAc, and that the other $\mathrm{TP}^{-}$'s formed in the more than two VMTP sequences cannot transfer any electrons to DCNB.

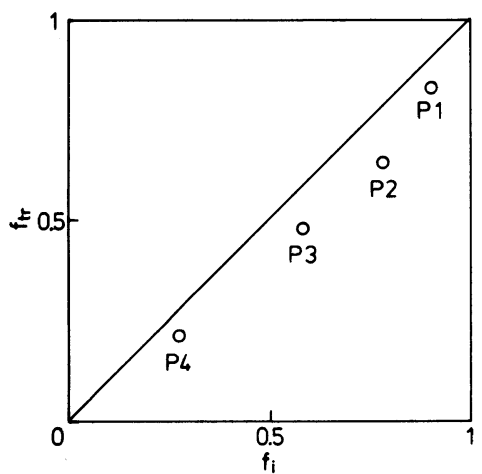

Figure 4. Transferable fraction $f_{\mathrm{tr}}$ versus isolated fraction $f_{i}$. 
As for $\mathrm{TP}^{-}$formed in the homopolymer PVMTP, the anion radical transfer to DCNB does not occur at all. It is stabilized by interactions among more than two TP chromophores.

\section{Comparison with Dimer Model Compounds}

To compare the diad sequence of VMTP in the copolymer with the dimer model compounds, we investigated again the anion radical transfer from the $\mathrm{TP}^{-}$of dimer model compounds and DMTP to the acceptor DCNB and determined $k_{\mathrm{tr}}$ more exactly by the initial transient absorption decay of $\mathrm{TP}^{-}$at $530 \mathrm{~nm}$ in the time region $(<1 \mu \mathrm{s})$ where the recombination process can be neglected. The measured rate constants are as follows, $1.2 \times$ $10^{9} \mathrm{M}^{-1} \mathrm{~s}^{-1}$ for DMTP, $1.0 \times 10^{9} \mathrm{M}^{-1} \mathrm{~s}^{-1}$ for 2,4-MTP, and 7.2 $\times 10^{8} \mathrm{M}^{-1} \mathrm{~s}^{-1}$ for 1,3-MTP. The anion radicals of dimer model compounds transfer their electron to DCNB more slowly than that of DMTP by the stabilization through interactions between the neighboring chromophores. Furthermore, the stability of 1,3-MTP is stronger than that of 2,4-MTP, which can be explained by the difference in the conformation of the dimer model compounds, i.e., 1,3-MTP is easier to take sandwich-like conformation than 2,4-MTP.

On the other hand, the anion radical $\mathrm{TP}^{-}$ formed in the sequence, VAc-VMTP-VMTPVAc on the copolymer does not transfer its electron to DCNB as described above. This suggests that $\mathrm{TP}^{-}$in the dimer sequence, VAcVMTP-VMTP-VAc on the copolymer is more stable than the dimer model compound. This large stabilization seems to be caused partly by the stronger neighboring interaction and partly by steric hindrance of the polymer chain to an approaching DCNB. In the latter case, it is plausible that the electron transfer in polymer systems occurs at a longer distance than in low-molecular compounds system since DCNB cannot approach the $\mathrm{TP}^{-}$by the steric hindrance. This reduces electron transfer rate constants. Figure 5 shows a summary of the

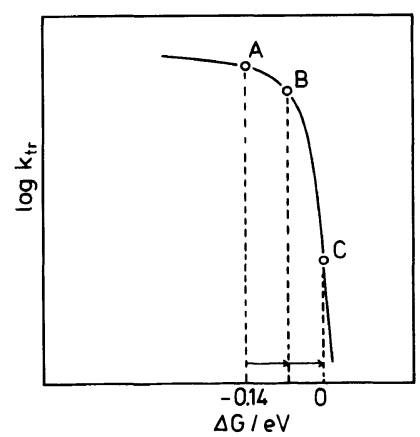

Figure 5. Relation between $k_{\mathrm{tr}}$ and free energy change in the anion radical transfer from $\mathrm{TP}^{-}$to DCNB.

above situation. The relation between $k_{\mathrm{tr}}$ and free energy change $(\Delta G)$ for the electron transfer is shown by the solid curve: $k_{\mathrm{tr}}$ decreases slowly with increase of $\Delta G$ in the exothermic region and drops rapidly at $c a . \Delta G=0 . .^{32}$ For monomer model compounds system, the free energy change $(\Delta G)$ of the anion radical transfer to DCNB is estimated to be $c a$. $-0.14 \mathrm{eV}$ from the difference of the reduction potentials of $\operatorname{DMTP}\left(E_{1 / 2}=-2.11 \mathrm{~V}\right)$ and $\operatorname{DCNB}\left(E_{1 / 2}=\right.$ $-1.97 \mathrm{~V})$. This case is represented by point $\mathrm{A}$. For the dimer model compound, $k_{\mathrm{tr}}$ becomes smaller than that of the monomer model compound by stabilization through interactions between the neighboring chromophores. The system is denoted by point $\mathrm{B}$. As for the anion radical in consecutive TP's in the copolymer, they are further stabilized by neighboring interactions or steric hindrance. This makes $\Delta G$ larger and the system may correspond to point C. In this case, even a slight increase of $\Delta G$ causes a large decrease of $k_{\mathrm{tr}}$ around $\Delta G=0$. As for the homopolymer, anion radical transfer was not observed in our experimental resolution; therefore, $\mathrm{TP}^{-}$in the homopolymer is estimated to be at least $0.1 \mathrm{eV}$ more stable than DMTP ${ }^{-}$.

\section{CONCLUSION}

The anion radical $\mathrm{TP}^{-}$formed in the copolymer of VMTP with VAc is stabilized depending on the location of the VMTP. That 
is, $\mathrm{TP}^{-}$isolated by a comonomer VAc in the copolymer transfers an electron to DCNB with a rate constant of $7.7 \times 10^{8} \mathrm{M}^{-1} \mathrm{~s}^{-1}$, whereas $\mathrm{TP}^{-}$formed in the VMTP sequences does not transfer an electron to DCNB; neither does the anion radical $\mathrm{TP}^{-}$in the homopolymer PVMTP transfer an electron to DCNB. This is partly due to neighboring interactions and partly due to the steric hindrance of the polymer chain to an approaching DCNB. The steric hindrance is considered to be one reason why $\mathrm{TP}^{-}$formed in the dimer sequence in the copolymer is more stable than the one in a dimer model compound.

\section{REFERENCES}

1. J. Guillet, "Polymer Photophysics and Photochemistry," Cambridge University Press, London, 1985.

2. M. Winnik, "Photophysical and Photochemical Tools in Polymer Science,” D. Reidel Publishing Co., Dordrecht, 1986.

3. D. Phillips and G. Rumbles, "Photochemistry and Photophysics in Polymers," N. S. Allen and W. Schnabel, Ed., Elsevier Applied Science Publishers Ltd., London, 1984, p 153.

4. W. Schnabel, "Developments in Polymer Photochemistry-3," N. S. Allen, Ed., Applied Science Publishers Ltd., London, 1982, p 237.

5. W. Klöpffer, "Organic Molecular Photophysics," J. B. Birks, Ed., John Wiley \& Sons, Inc., London, 1973, Chapter 7.

6. F. C. De Schryver, P. Collart, J. Vandendriessche, R. Goedeweeck, A. M. Swinnen, and M. Van der Auweraer, Acc. Chem. Res., 20, 159 (1987); J. Vandendriessche, P. Palmans, S. Toppet, N. Boens, F. C. De Schryver, and H. Masuhara, J. Am. Chem. Soc., 106, 8057 (1984).

7. W. Klöpffer, Ann. N. Y. Acad. Sci., 366, 373 (1981).

8. A. M. North and M. F. Treadaway, Eur. Polym. J., 9, 609 (1973); A. M. North and D. A. Ross, J. Polym. Sci. Symposium, 55, 259 (1976).

9. S. Ito, M. Yamamoto, and Y. Nishijima, Polym. J., 13, 791 (1981); S. Ito, K. Yamashita, M. Yamamoto, and Y. Nishijima, Chem. Phys. Lett., 117, 171 (1985).

10. S. Ito, M. Yamamoto, and Y. Nishijima, Bull. Chem. Soc. Jpn., 54, 35 (1981).

11. G. E. Johnson, J. Chem. Phys., 62, 4697 (1975); M. Yokoyama, T. Tamamura, M. Atsumi, M. Yoshimura, Y. Shirota, and H. Mikawa, Macromolecules, 8, 101 (1975); H. F. Kauffmann, W. Weixelbaumer, J. Buerbaumer, A. Schmoltner, and
O. F. Olaj, ibid., 18, 104 (1985).

12. H. Masuhara, S. Ohwada, N. Mataga, A. Itaya, K. Okamoto, and S. Kusabayashi, J. Phys. Chem., 84, 2363 (1980); H. Masuhara, N. Tamai, K. Inoue, and N. Mataga, Chem. Phys. Lett., 91, 109 (1982).

13. U. Lachish, D. J. Williams, and R. W. Anderson, Chem. Phys. Lett., 65, 574 (1979); U. Lachish, R. W. Anderson, and D. J. Williams, Macromolecules, 13, 1143 (1980).

14. I. C. Lewis and L. S. Singer, J. Chem. Phys., 43, 2712 (1965).

15. A. Carrington and A. D. McLachlan, "Introduction to Magnetic Resonance," Harper \& Row, Publishers Inc., New York, N. Y., 1967, Chapter 8.

16. H. D. Roth, "Chemically Induced Magnetic Polarization," L. T. Muus, P. W. Atkins, K. A. McLauchlan, and J. B. Pedersen, Ed., NATO-ASI, D. Reidel Pub. Co., Dordrecht, 1977, p 39.

17. B. Badger, B. Brocklehurst, and R. D. Russell, Chem. Phys. Lett., 1, 122 (1967); B. Badger and B. Brocklehurst, Trans. Faraday Soc., 65, 2576 (1969); B. Badger and B. Brocklehurst, ibid., 65, 2582 (1969); B. Badger and B. Brocklehurst, ibid., 65, 2588 (1969).

18. R. E. Bühler and W. Fund, J. Phys. Chem., 79, 2098 (1975).

19. A. Kira, M. Imamura, and T. Shida, J. Phys. Chem., 80, 1445 (1976); A. Kira and M. Imamura, ibid., 83, 2267 (1979).

20. S. Irie, H. Horii, and M. Irie, Macromolecules, $\mathbf{1 3}$, 1355 (1980); S. Irie and M. Irie, ibid., 19, 2182 (1986).

21. A. Kira, S. Arai, and M. Imamura, J. Chem. Phys., 54, 4890 (1971); A. Kira, S. Arai, and M. Imamura, J. Phys. Chem., 76, 1119 (1972).

22. M. Washio, S. Tagawa, and Y. Tabata, Polm. J., 13, 935 (1981).

23. H. Masuhara, N. Tamai, N. Mataga, F. C. De Schryver, and J. Vandendriessche, J. Am. Chem. Soc., 105, 7256 (1983); H. Masuhara, K. Yamamoto, N. Tamai, K. Inoue, and N. Mataga, J. Phys. Chem., 88, 3971 (1984).

24. S. Tazuke, H. L. Yuan, Y. Iwaya, and K. Sato, Macromolecules, 14, 267 (1981); K. Iwai, M. Furue, S. Nozakura, Y. Shirota, and H. Mikawa, Polym. J., 12, 97 (1980).

25. Y. Tsujii, A. Tsuchida, M. Yamamoto, and Y. Nishijima, Macromolecules, 21, 665 (1988).

26. T. Shida and S. Iwata, J. Chem. Phys., 56, 2858 (1972).

27. S. Arai, A. Kira, and M. Imamura, J. Phys. Chem., 81, 110 (1977).

28. A. Tsuchida, M. Yamamoto, and Y. Nishijima, $J$. Phys. Chem., 88, 5062 (1984); A. Tsuchida, N. Masuda, M. Yamamoto, and Y. Nishijima, Macromolecules, 19, 1299 (1986).

29. R. L. Adilman, J. Am. Chem. Soc., 71, 1057 (1949).

30. M. Fineman and S. D. Ross, J. Polym. Sci., 5, 259 (1950). 
31. G. E. Ham, "Copolymerization," G. E. Ham, Ed., High Polymers Vol. XVIII; Interscience Publishers, John Wiley \& Sons, Inc., New York, N. Y., 1964,
Chapter 1.

32. D. Rehm and A. Weller, Israel J. Chem., 8, 259 (1970). 